\title{
A NEW MEMBER OF THE COLLOQUIUM
}

Professor H. Zins of the University of Lublin, Poland, has just joined the Department of History at Laurentian University, Sudbury. Professor Zins has worked on sixteenth-century economic history and has written a book on Anglo-Polish trade in the Baltic Sea. He has recently completed a study of the members of the cathedral chapter at Frauenberg, where Copernicus was canon from 1506 to 1543.

\section{NEWS OF THE RENA ISSANCE SOCIETY OF AMERICA}

The Toronto Renaissance and Reformation Colloquium was received at the eighteenth regional conference in the Renaissance Society of America as its annual meeting in New York this January. The Society is celebrating its 25th anniversary. All readers of the Bulletin who are interested in joining the Society as individuals and receiving the Renaissance Quarterly and the annual Stuaies in the Renaissance may send their annual dues of $\$ 8.00$ (US) to the Renaissance Society of America, Inc., 1161 Amsterdam Avenue, New York, N.Y. 10027.

\section{UN NOUVEAU_CENTRE D'ETUDES DE IA RENAISSANCE A L'UNIVERSITE DE SHERBROOKE}

Le Toronto Renaissance and Reformation Colloquium a reçu avec plaisir la nourelle de la fondation d'un Centre d'Etudes de la Renaissance à L'Université de Sherbrooke. On espère qu'il y aura beaucoup de contactes entre les spécialistes de la Renaissance à Toronto et à Sherbrooke。 Nous publions ici un communiqué du. professeur J.M.DE BUJANDA, directeur du Centre:

Fondé en 1968 grâce à une subvention du Conseil de la Recherche du Québec, le Centre d'études de la Renaissance de l'Université de Sherbrooke a comme but:

a) Rassembler une documentation aussi complète que possible sur la civilisation et Ia culture de la Renaissance;

b) Promouvoir et coordonner aux niveaux supérieurs la recherche et l'enseignement qui concernent cette période;

c) Promouvoir, en se servant des moyens les plus modernes, la recherche et le travail interdisciplinaires;

d) Favoriser les contacts et les échanges de professeurs et d'étudiants avec les autres centres similaires au Canada et à l'étranger;

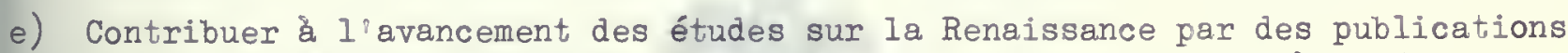
scientifiques et par l'organisation des colloques ou des congrès d'étude.

Le Centre, qui est conçu comme un organisme interdisciplinaire, rassemble des professeurs qui ont des projets de recherche sur cette époque et des étudiants, au niveau de la maitrise et du doctorat, qui ont choise la période de la Renaissance comme spécialisation. 